\title{
Evolution morphologique du lit de la Loire en aval d'un ouvrage réçent.
}

P. Fattal

: Institut de Géographie - Université deNantes.

Y. Delanoë, S. André : Laboratoire de Mécanique et Géomécanique Université de Nantes.

Résumé

Depuis 1992, le Laboratoire de Mécanique et Géomécanique et l'Institut de Géographie ont entrepris et poursuivent des travaux de recherche sur le site du bras de la Madeleine en Loire estuarienne, au coeur de la ville de Nantes.

Ces investigations sont destinées à mieux y appréhender les phénomènes dynamiques tant au niveau morphologique, sédimentologique que courantologique. En effet, les aménagements successifs (dragages, entretien du chenal de navigation, création d'un bassin à marée, extractions de granulats et constructions d'ouvrages d'art) ont considérablement modifié le milieu et ce laboratoire " grandeur nature " s'avère riche en enseignements.

Parmi nos préoccupations, l'étude des affouillements en aqal des piles de ponts et en l'occurence de celles du pont Anne de Bretagne a particulièrement retenu notre attention. Si ce dernier construit en 1973 ne comporte pas de risques majeurs puisque fondé au rocher, il permet de confirmer ou d'infirmer des essais de laboratoire et pourquoi pas à terme de déboucher sur une modélisation applicable à d'autres sites ; l'intérêt étant d'estimer les risques d'affouillement liés à chaque ouvrage.

\section{1 - Site étudié}

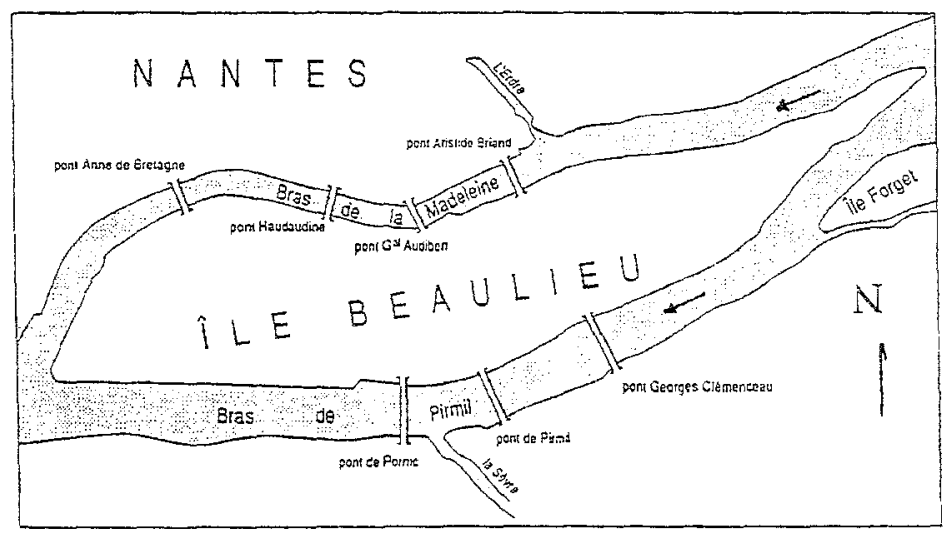

figure 1 : carte de localisation

Des levés bathymétriques ont été effectués dans le bras de la Madeleine par le Port Autonome de Nantes - St Nazaire (PANSN) et nos laboratoires : janvier 92 (PANSN) ; mai 93 (Université) ; avril , mai , juillet et septembre 94 (Université, PANSN); mai 95 (Université). 
Ce site remplit différents critères : il est facile d'accès; aucune intervention humaine n'a été effectuée dans les fosses résultant de l'affouillement; celles-ci se sont considérablement creusées entre 92 et 95 (notamment la fosse située en aval de la pile proche de la rive droite) et enfin, il correspond à une partie des investigations de recherches menées dans les bras enserrés par la ville.

En effet, nos recherches élargies ont pour but:

- de suivre l'évolution du fond notamment depuis la fin des extractions de granulats,

- de comprendre le comportement du bouchon vaseux et l'évolution du front de salinité,

- de mesurer limpact des aménagements existants sur l'hydraulique et les ouvrages,

- de modéliser l'estuaire afin de déterminer les conséquences que peuvent entraîner des aménagements sur l'hydraulique.

\section{2 - Les affouillements et la dymamique du bras de la Madeleine}

\section{2 - 1 - Hydraulique du bras de la Madeleine}

Il était jusqu'à présent communément admis que le débit de la loire était deux fois plus important dans le bras de Pirmil que dans celui de la Madeleine. Des mesures de vérifications effectuées parallèlement à cette étude, durant l'été 94 et le printemps 95 ont conduit à réviser cette estimation. Les résultats ont montré que le partage se fait pratiquement à egalité entre les deux bras (47\% pour le bras de la Madeleine contre 53\% pour celui de Pirmil)(8).

En conséquence, et en s'appuyant sur les données de débits de la Loire fournis par la station de Montjean, on estime que le débit dans le bras de la Madeleine (Q Mad $\mathrm{m}^{3} / \mathrm{s}$ ) est égal à la moitié du débit fluvial (Q) (voir tableau des débits).

\begin{tabular}{|c|c|c|}
\hline & $\begin{array}{c}\text { Q } \mathbf{~ m}^{\mathbf{3}} / \mathrm{s} \\
\text { (module interannuel) }\end{array}$ & $\begin{array}{c}\text { Q Mad } \mathbf{~ m}^{\mathbf{3}} / \mathbf{s} \\
\text { (Madeleine) }\end{array}$ \\
\hline 1992 & 685 & 342 \\
\hline 1993 & 649 & 324 \\
\hline 1994 & 1208 & 604 \\
\hline $1995^{*}$ & 2838 & 1419 \\
\hline
\end{tabular}

(*) : valeurs du premier trimestre 95

tableau 1 : Les débits de la Loire à Montjean (estimations dans le bras de la Madeleine)

Enfin, on observe que sur les quatre années considérées, les deux dernières enregistrent des crues non négligeables favorisant la mobilité des sédiments.

\section{2 - 2 - Caractéristiques du lit : mobilité des sédiments}

Les données sédimentologiques dans le secteur sont très variables. D'une année et d'une saison à l'autre, on voit apparaître du sable fin ou grossier (93 - 07/94) mais aussi le bouchon vaseux et la crème de vase $(92$ - 09/94). (Fattal,1993 (5).

De ce fait, nous avons délibérement choisi de ne pas réaliser une carte sédimentologique qui ne pourrait être représentative que d'une période déterminée avec des conditions hydrologiques spécifiques. 
Les calculs ont été faits pour un sable fin, matériau le plus communément trouvé dans la zone. A cet effet, nous avons considéré que le diamètre médian du sédiment est généralement : $0,21 \mathrm{~mm}<\mathrm{d}<0,44 \mathrm{~mm}$.

De plus, pendant la période de campagne, nous n'avons aucune trace de la crème de vase, ni du bouchon vaseux.

Les particules en présence sont soumises à un charriage dès lors que la force motrice due au courant ( $\mathrm{Fm}$ ) est supérieure à la force de frottement du grain sur le sol (Ff) ou encore que la vitesse moyenne de l'écoulement de l'eau (U) est supérieure à la vitesse critique d'entraînement du matériau (Uc).

On a:

$$
U_{c}^{2}=K_{1} \delta g d \text { avec } K_{1}=\frac{4}{3} * \frac{f}{C f} \quad \text { (sur fond plat) }
$$

( $\delta$ : densité apparente du sédiment; g: accélération de la pesanteur; d: diamètre médian du grain; f: coef. de frottement; Cf: coef. de forme).

Le coefficient de frottement $f$ peut être obtenu par la formule de Ramette (14):

$$
f=\frac{26.4}{d^{1 / 6}}\left(\frac{d}{H}\right)^{1 / 24}
$$

pour une profondeur d'eau moyenne $\mathrm{H}=11,5 \mathrm{~m}$. une densité apparente $0,83<\delta<1,24$ et pour l'étendue granulométrique considérée on obtient :

$$
0,39 \mathrm{~m} / \mathrm{s}<\mathrm{U}_{\mathrm{c}}<0,66 \mathrm{~m} / \mathrm{s}
$$

Par ailleurs et afin de comparer $U$ à $U_{C}$, nous avons calculé $U=\frac{Q M a d}{S}$ (QMad: débit moyen pendant la crue $\left\{\mathrm{Qmad}=1419 \mathrm{~m}^{3} / \mathrm{s}\right\}$; S: section moyenne à l'aval du pont Anne de Bretagne $\left.\left\{S=\mathrm{L} \times \mathrm{H}=135 \times 11,5=1452 \mathrm{~m}^{2}\right\}\right)$.

On obtient : $\mathrm{U}=\mathbf{0 , 9 8} \mathrm{m} / \mathrm{s}$.

En conséquence, pendant la crue, mais aussi hors crues (mai 95, pour un débit moyen de $413 \mathrm{~m}^{3} / \mathrm{s}$ ), U> Uc. Les sédiments sont donc charriés.

\section{2 - 3 - Affouillement en aval du pont A. de Bretagne : de la théorie à la pratique}

Un affouillement est une mobilisation du sol sous l'action d'un courant. En effet, lorsque $\mathrm{U}>\mathrm{U}_{\mathrm{C}}$, deux possibilités se présentent:

- soit le lit est érodé et le matériau arraché

- soit l'épaisseur du lit mobilisé perd ses propriétés mécaniques.

Dans ces deux cas on parle de sols affouillés, quelque soit la nature du sol (frottant, cohérent ou roches). Les conséquences ne sont donc pas négligeables et l'affouillement peut aller jusqu'à la destabilisation d'un ouvrage d'art.

Dans cette étude, nous nous cantonnons aux sols non cohésifs.

Nous distinguons pour le bras de la Madeleine, trois types d'affouillements:

- général

- local

- dú à l'effet de seuil

$2-3-1$ - llaffouillement général

Dans le bras de la Madeleine, nous dénombrons plusieurs causes susceptibles d'engendrer cette érosion:

- l'effet de la crue de 1995 , 
- un retrécissement généré par la présence d'une barge en aval du pont $\mathrm{A}$. de Breragne,

- l'allure générale du bras et sa forme coudée dans le secteur,

- les activités de dragages (aujourd'hui, théoriquement abandonnées).

\subsubsection{L'effet de crue :}

Soit y la profondeur des fonds mobilisés pendant la crue, à partir de la surface moyenne de l'eau $(\mathrm{m})$. Soit $q$ le débit dans le iit par unité de largeur

( $q=\frac{\text { QMad́d }}{B}$ (QMad : débit pendant la crue; B: largeur moyenne du lit ).

On applique alors la formule de Vigoureux et Laraichi (9), en tenant compte de d (diamètre médian du grain en $\mathrm{m}$.)

$$
y=0,73 \frac{q^{2 / 3}}{d^{1 / 6}}=13,4 \mathrm{~m} \text { soit }-16,13 \mathrm{~m} \mathrm{IGN}
$$

La fondation de la pile, rive droite du pont se situe à $-11,8 \mathrm{~m}$ IGN à même la roche. Après la crue de 95 , le lit de sable autour de cette pile est donc totalement mobilisé.

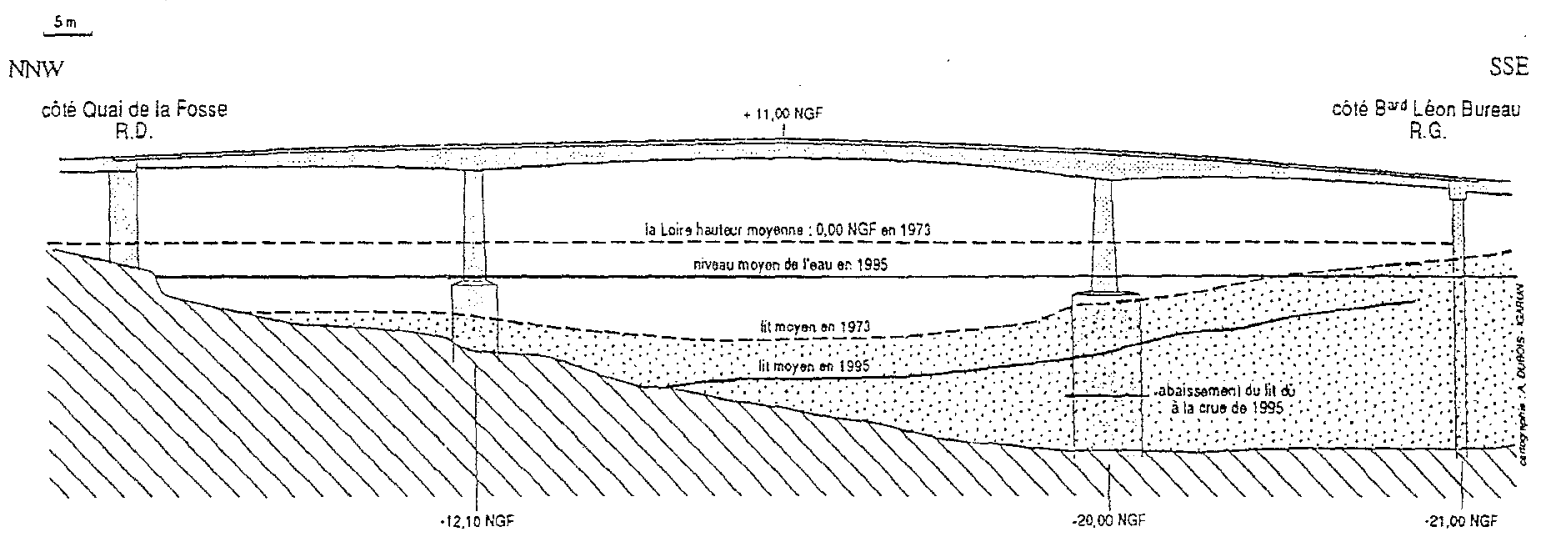

figure 2 : le lit de la Loire et l'effet de crue (document Mairie)

2.3.1.2. L'effet de retrécissement:

Soit e : l'épaisseur du lit affouillé (m). On peut dans le cas d'un pincement également appliquer la formule ci- dessus, en tenant compte de $\mathrm{H}$ (hauteur d'eau moyenne du lit en $\mathrm{m}$ ).

Le calcul donne : $e=\mathbf{2 , 4}$ mètres.

Afin de vérifier le calcul, nous avons comparé les bathymétries des deux dernières années et obtenu un affouillement $\mathrm{e}=\mathbf{2 , 5}$ mètres.

En conséquence, nous pouvons considérer que la méthode de Vigoureux et Laraichi donne une estimation correcte de la profondeur de l'affouillement dû à un retrécissement dit court (la longueur de la barge (L) est de $79,5 \mathrm{~m}$, alors que la largeur du lit (B) avoisine les $150 \mathrm{~m})$. Un retrécissement long $(\mathrm{L}>\mathrm{B})$ aurait entraîné l'application d'une autre formule tenant compte de la profondeur moyenne et de la largeur du retrécissement. 


\subsubsection{Les autres causes:}

L'effet de coude du bras (figure 3) a pour conséquence de concentrer le courant dans la partie extérieure du coude et donc vers la rive droite du lit (voir paragraphe 3).

De fait, les affouillements en aval de la pile à proximité de la rive droite sont plus marqués que ceux situés en aval de la pile gauche.

Par ailleurs, les dragages ont cessé depuis 1992 mais ils ont pour conséquence d'avoir contribué à l'abaissement du lit.

Malheureusement, nous ne disposons pas d'informations suffisantes pour estimer l'effet de ces deux dernières causes sur l'affouillement général.

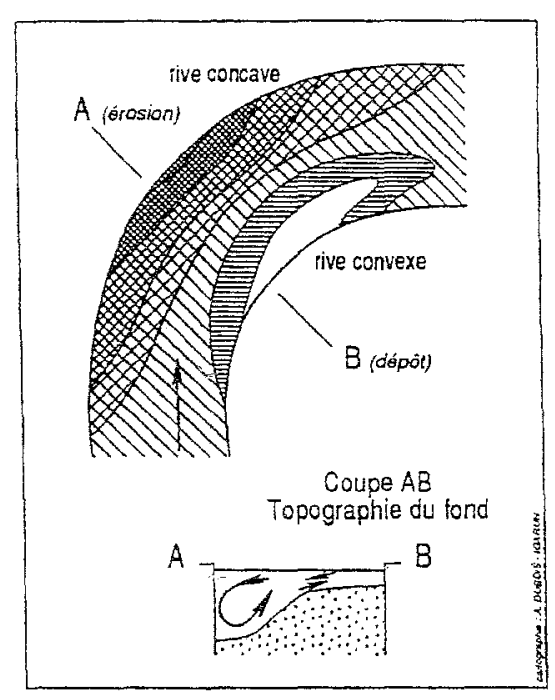

\section{figure 3 :}

érosion et sédimentation dans un coude

\section{$2-3-2$ - l'affouillement local}

Au fond, la pile crée une turbulence de l'écoulement. Cet agent d'érosion se manifeste en amont de la pile par un système de vortex en forme de fer à cheval; en aval, il engendre un vortex de sillage (figure 4). Ces vortex sont de véritables aspirateurs, arrachant les grains autour de la pile pour les transporter et les déposer au delà, en aval du courant.

Les vortex dus au sillage doivent théoriquement s'appliquer aux deux piles du pont. En réalité, celui de la rive droite est bien plus marqué que celui de l'autre rive. Certains élèments d'explications proviennent de l'effet de coude et de seuil (voir paragraphe 3).

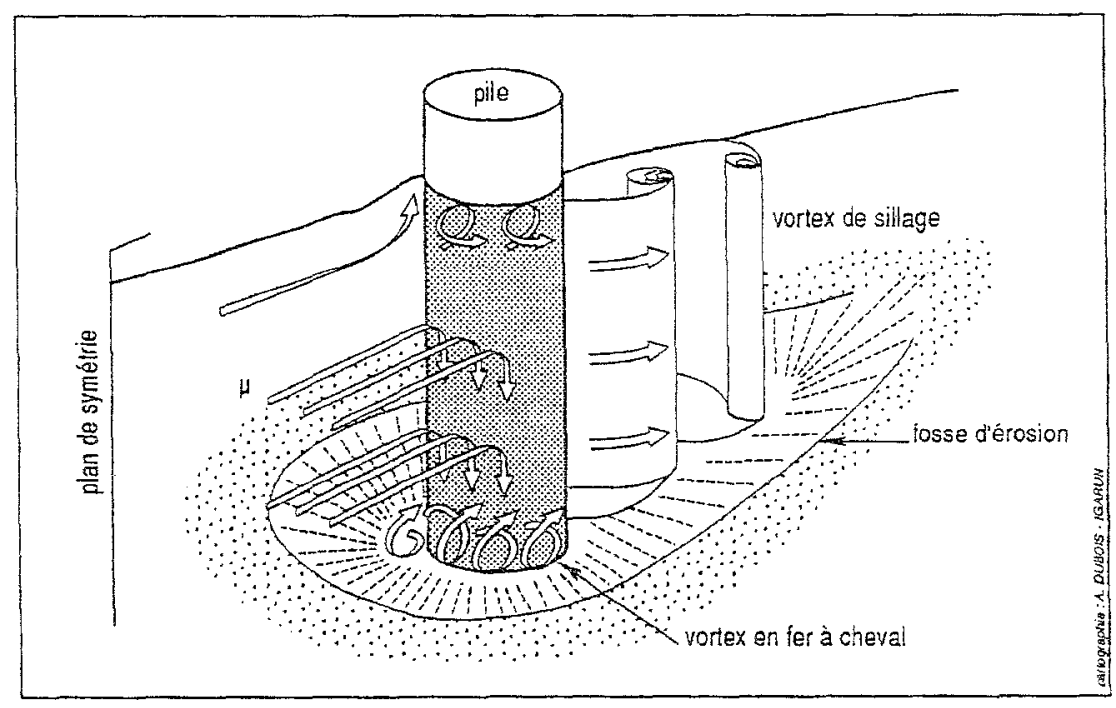

figure 4 : structure de l'écoulement autour d'une pile de pont 
$2-3-3-$ L'affouillement dû à l'effet de seuil

Lorsque le courant

franchit un seuil, l'écoulement stable dissipe de l'énergie au niveau du ressaut, il devient torrentiel et il se crée, en aval, une fosse quasi-ellipique (figure 5).

Sur la pente, en aval de l'obstacle les grains sont arrachés puis tansportés. Il faut par ailleurs noter qu'il existe un courant de retour (aval vers amont) dont on maîtrise mal l'effet sur la fosse.

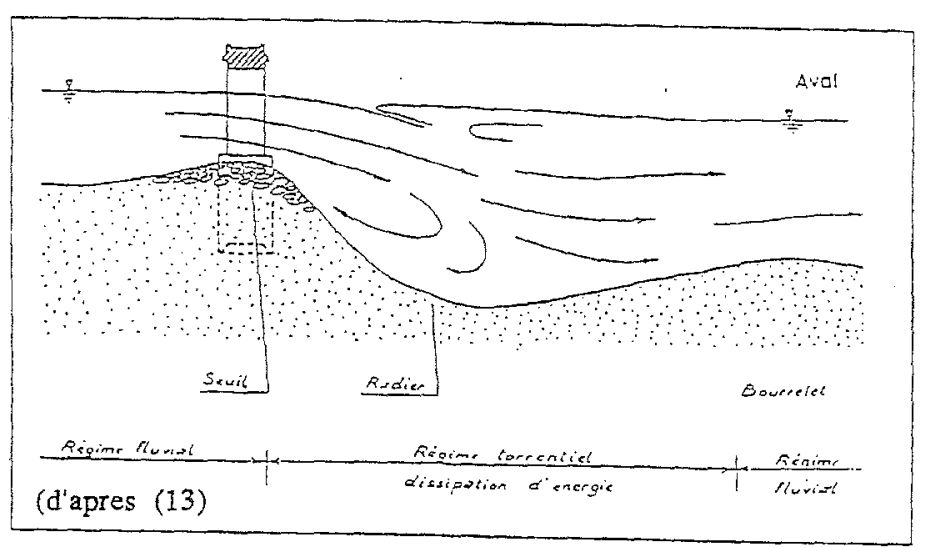

figure 5 : écoulement au niveau d'un seuil (d'après (13)

Dans le bras de la Madeleine, un bateau d'une quarantaine de mètres est coulé, le long de la rive droite à $20 \mathrm{~m}$ en amont du pont Anne de Bretagne. Cette importante épave constitue un seuil artificiel équivalent d'un barrage submergé ou d'un dénivelé naturel. On peut donc appliquer la formule de Breusers (3):

$$
\frac{d_{s}}{H}=0,11 \times \delta^{-0,646} \times H^{-0,76} \times\left(\alpha U-U_{c}\right)^{1,634} \times t^{0,38}
$$

En prenant une profondeur $\mathrm{H}=11,5 \mathrm{~m}$, le débit moyen de la crue de 1995 (1419 $\mathrm{m}^{3} / \mathrm{s}$ ), une densité apparente $\delta=0,83$, un coefficient de forme $\alpha=2,5$ et une durée $\mathrm{t}=3,4$ ans (de 93 à 95) nous obtenons une évolution de la profondeur :

$$
\mathrm{d}_{\mathrm{s}}=1,07 \mathrm{~m} \text {. }
$$

Cette valeur est nettement inférieure à l'observation sur le terrain $(2 \mathrm{~m}$ d'affouillement po⿺𠃊r la même période). Cela ne remet pas en cause la validité de la formule expérimentale de Breusers puisque l'effet de seuil n'est qu'un des élèments intervenant dans l'affouillement.

\section{3 - Evolution de la fosse}

\section{3 - 1 - Situation générale (figure 6)}

La morphologie du bras de la Madeleine se présente de la manière suivante:

- une fosse marquée en aval de la pile droite du pont Anne de Bretagne qui se prolonge jusqu'au droit du bâtiment du Port Autonome; alors que celle de la pile gauche est beaucoup moins franche (voir figure 7);

- une zone de sédimentation le long de la rive gauche, 50 mètres en aval de la culée gauche et en contrebas du quai des Antilles. Les sédiments déposés dans le secteur sont de type vaseux ou vase sableuse. Par fort coefficients de marée, ils découvrent à basse-mer et on peut observer leur profil convexe à pente marquée. 
Bras de la Madeleine : évolution des fonds 1992-1995

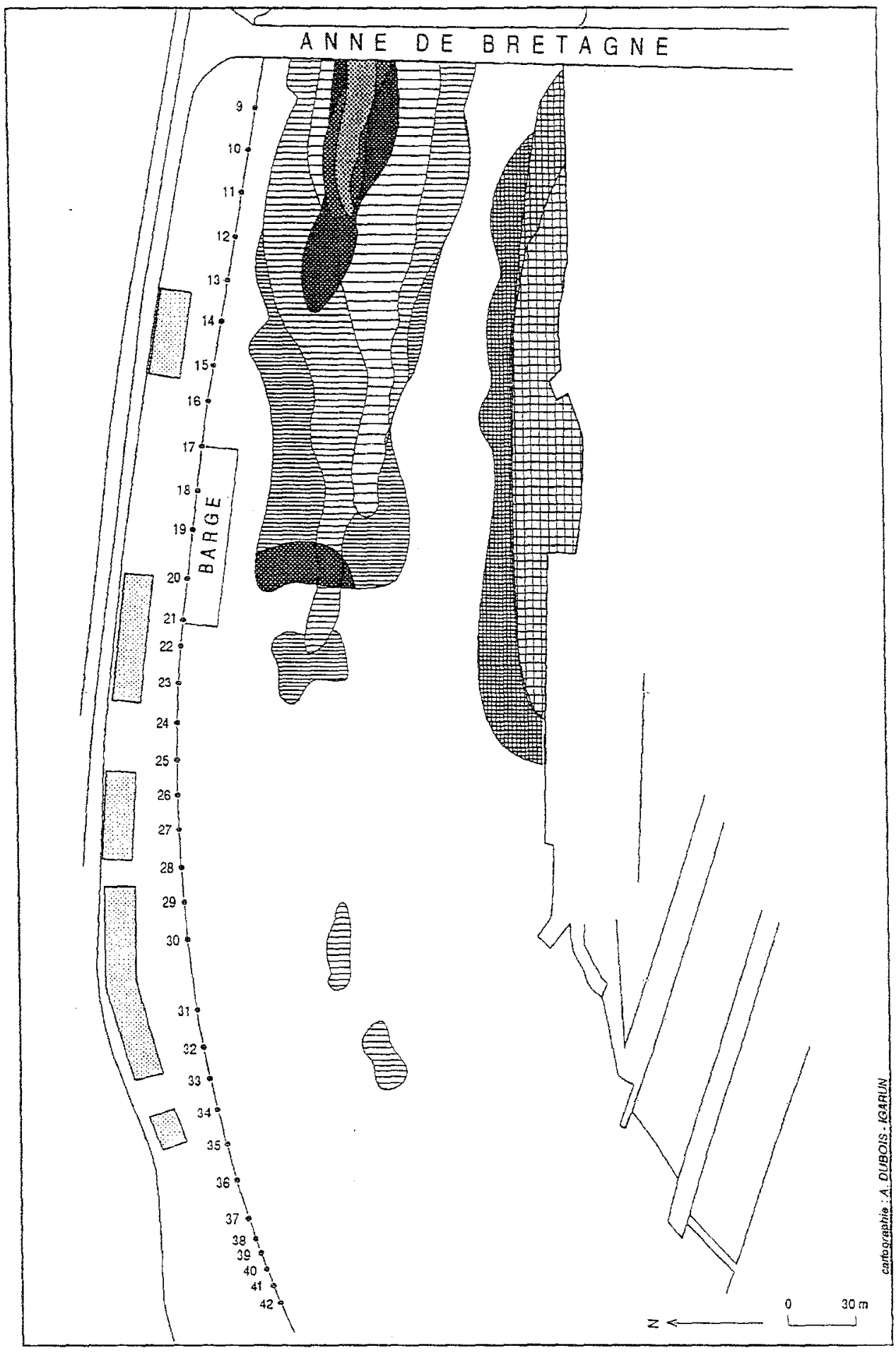

\begin{tabular}{|c|c|c|c|}
\hline & fond de $-6,5$ à $-7 \mathrm{~m} C . M$ & fond de $-4,5$ à $-6,5 \mathrm{~m} \mathrm{C.M}$ & fond au-dessus du O C.M \\
\hline janvier 1992 & א. & $E$ & \pm \\
\hline avil 1994 & & $\equiv$ & 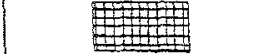 \\
\hline mai 1995 & & $=$ & W \\
\hline
\end{tabular}


Bras de la Madeleine : évolution 1992-1995
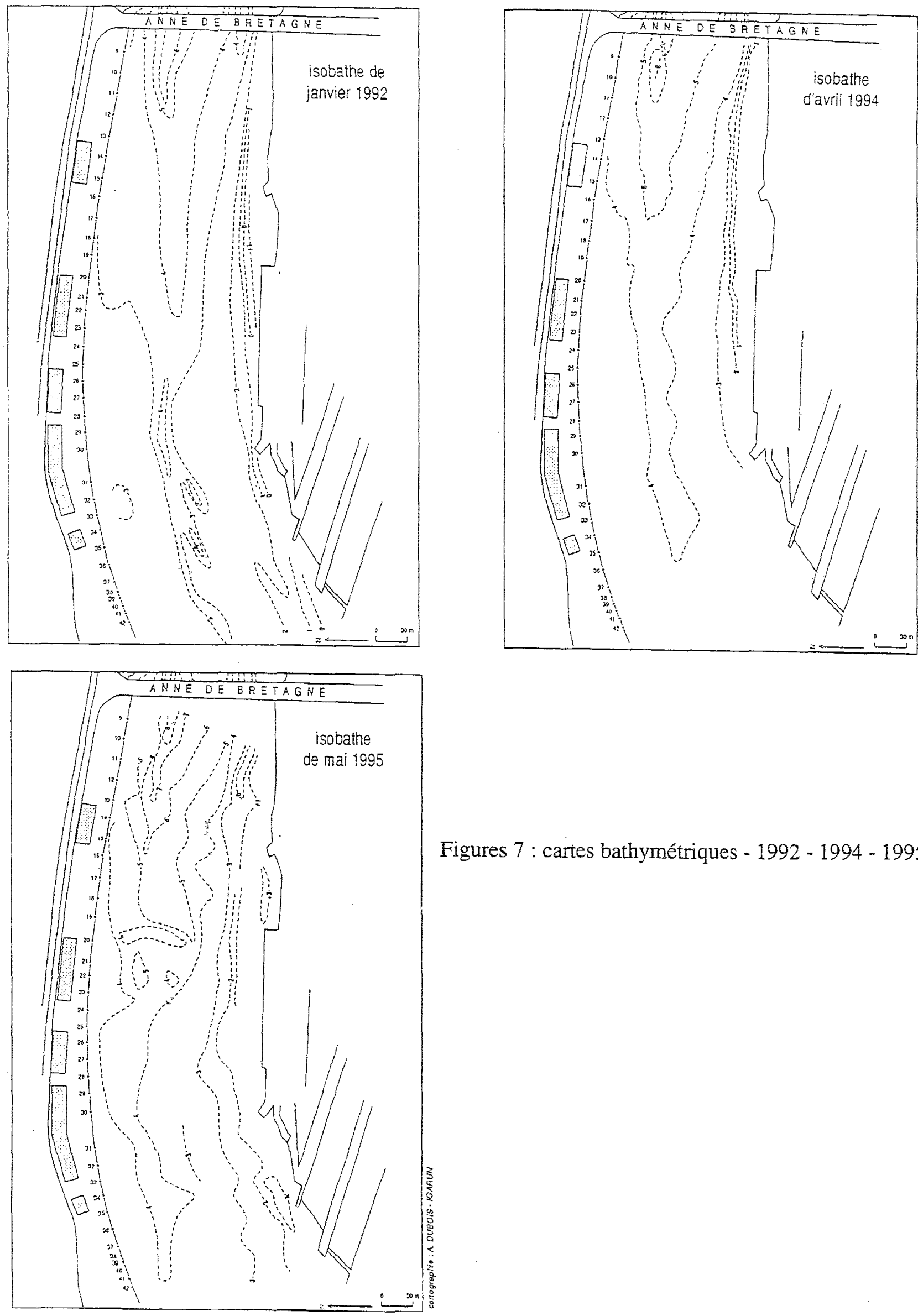

Figures 7 : cartes bathymétriques - 1992 - 1994 - 1995. 


\section{3 - 2 - Vers un élargissement et un approfondissement}

\section{3 - 2 - 1 - Evolution de 1992 à 1995}

Les cartes en isobathes des années 92 à 95 (figures 7) révèlent une évolution de la fosse (analyse de la partie aval de la rive droite) qui se manifeste par un creusement et un élargissement. En effet, à partir de 94, le chenal s'élargit. En 1995, il se décale d'approximativement 25 mètres, vers le quai de la Fosse situé sur la rive droite de la Loire. Juste en aval de la pile la plus marquée, on observe l'évolution du creusement en suivant le contour des isobathes allant de -6 CM. (en 92) à -8 CM. (en 94 et 95). On peut par ailleurs observer que la fosse est nettement délimitée en 92 par l'isobathe $-3 \mathrm{CM}$., alors qu'en 94 et 95 c'est la courbe $-4 \mathrm{CM}$. qui la définit.

Sur la rive gauche l'isobathe $-3 \mathrm{CM}$. regresse de 92 à 94 puis progresse vers le lit à nouveau de 94 à 95 . Dans ce secteur, les dépôts au dessus du O CM. régressent de 92 à 94 puis s'intensifient en 1995. II apparaît donc des variations de périodes de sédimentations et d'érosions. Ainsi, la crue de 95 érode le fond du lit et la fosse, étalant ses matières en suspension sur la rive gauche où l'effet du courant est le plus faible.

De manière synthétique, les caractéristiques de la fosse se présentent de la forme suivante :

\begin{tabular}{|c|c|c|c|c|}
\hline Année & $\mathbb{I}_{\mathbf{S}}(\mathbf{m})$ & $\mathbf{L}_{\mathbf{S}}(\mathbf{m})$ & $\begin{array}{c}\mathrm{d}_{\mathbf{S}}(\mathbf{m} \text { en } \\
\mathbf{C M})\end{array}$ & $\mathbf{V}_{\mathbf{S}}\left(\mathbf{m}^{3}\right)$ \\
\hline 1992 & 55,5 & 207 & 6,9 & $3.1010^{4}$ \\
\hline 1994 & 88,5 & 271,5 & 8,1 & $7,6.10^{4}$ \\
\hline 1995 & 84 & 294 & 8,9 & $8,6.10^{4}$ \\
\hline
\end{tabular}

tableau 2 : caractéristiques de la fosse

$1_{S}:$ largeur maximale de la fosse d'affouillement,

$\mathrm{L}_{S}$ : longueur maximale de la fosse d'affouillement,

$\mathrm{d}_{\mathrm{S}}$ : profondeur maximale de l'affouillement,

$\mathrm{V}_{\mathrm{S}}$ : volume de la fosse d'affouillement considérée comme elliptique (base du calcul).

$$
V_{s}=\frac{1}{2} \pi \times \frac{L_{s}}{2} \times \frac{l_{s}}{2} \times d_{s}
$$

ceci nous conduit au tableau de synthèse suivant:

\begin{tabular}{|l|c|}
\hline Progression maximale vers l'aval & $87 \mathrm{~m}$. en 3,4 ans \\
\hline Progression maximale en largeur & $30 \mathrm{~m}$. en 3,4 ans \\
\hline Surcreusement maximal & $2 \mathrm{~m}$ en 3,4 ans \\
\hline $\begin{array}{l}\text { Evaluation du volume de sédiments } \\
\text { arrachés }\end{array}$ & $5,5.10^{4} \mathrm{~m}^{3}$ en 3,4 ans \\
\hline
\end{tabular}

tableau 3 : synthèse 


\section{$3-2$ - 2 - Rythmes de l'évolution}

A partir des données de sondage nous avons tenté de définit des rythmes d'évolution, on obtient de janvier 1992 à avril 1994 un creusement de $0,52 \mathrm{~m}$ par an correspondant à un rythme d'érosion de $2 * 10^{4} \mathrm{~m}^{3} / \mathrm{an}$. De mai 1994 à mai 1995 , le creusement est de $0,8 \mathrm{~m}$ et le volume érodé de $1 * 10^{4} \mathrm{~m}^{3}$.

En conséquence, on constate que la fosse s'est creusée de manière plus notable de 94 à 95 que de 92 à 94 . Par contre le volume global du sédiment arraché est plus important de 92 à 94 . C'est pendant cette période que l'élargissement est le plus notable (voir figure ci-contre).

\subsubsection{Evolution}

Afin d'évaluer les cubatures des déblais et remblais dans le secteur, nous avons digitalisé les fonds bathymétriques sur MICROSTATION puis transféré les bases de données sur ARC INFO. Le résultat nous permet, d'une part de comparer les volumes à ceux calculés. Il apparaît que les volumes sont tout-à-fait comparables (Fattal, 1996 (7)).

\section{3 - 2 - 3 - Sunthèse des modes de développement de la fosse en aval du Pont A. de} Bretagne

Les causes de l'affouillement sont donc:

- les crues successives,

- l'effet de coude,

- les interventions humaines

. dragages

- retrécissement du lit

. seuils artificiels

La crue de janvier 95, justifie l'évolution importante. Toute augmentation du débit amène à un abaissement des fonds, du fait de la migration des sédiments mobilisables. De 94 à 95 , cet affouillement est plus marqué que de 92 à 94 .

Dans un coude, lécoulement n'est pas uniforme. L'érosion affecte la rive concave et les sédiments transportés peuvent se déposer sur la rive convexe. En effet, un étude menée en 93, sur les quais de la rive droite (Fattal, 1993 (5)) a montré que ces derniers étaient sensiblement dégradés dans le secteur le plus courbé.

La figure 3 montre que le modèle de l'écoulement dans le coude est complexe, puisqu'il se forme un écoulement secondaire, prés du fond, qui est en spirale. Dans le détail, le molvement général dans le coude s'accompagne d'un écoulement secondaire transversal au niveau du fond.

Par contre, l'effet de coude ne modifie pas les lignes d'eau en surface. Aussi, le transport des particules en suspension ne devrait pas être plus important sur la rive droite. Ceci est confirmé par les résultats des prélèvements de MES des campagnes successives.

Les dragages importants des années 70 à 90 ont conduit à un abaissement général des fonds d'environ 4 mètres. Aujourd'hui, ces prélèvements ont cessé mais le lit modifié dans sa morphologie doit à nouveau trouver son profil d'équilibre. Tant que celui-ci n'est pas atteint, le fond est continuellement mobilisé.

"Le retrécissement" récent du lit dû à la barge amarrée au niveau du bollard 17 ; 215 mètres à l'aval du pont entraîne une concentration et donc un augmentation de la vitesse du courant. La conséquence directe est la présence d'un affouillement localisé au niveau de la barge.

"Le seuil" dû à l'épave modifie l'écoulement, les sédiments transportés trouvent plus loin l'obstacle de la pile, pouvant entraîner la formation d'une butte qui ferait elle-même effet de seuil. Ainsi, dans le secteur d'étude il existe une conjonction de 
phénomènes qui tend à expliquer le fort et rapide creusement constaté en aval de la pile droite. Ceci permet aussi, de justifier que l'affouillement ne soit pas aussi significatif en aval de la pile gauche.

\section{4- Analyse critique des résultats}

Ce travail marque une étape dans la connaissance du milieu. De nombreuses autres sont à venir et elles nécessiteront, pour le cas de l'affouillement des campagnes d'investigation complémentaires dans les domaines suivants:

- Mesure de la pression interstitielle dans la fosse pour comprendre comment évoluent les propriétés mécaniques du sol,

- Mesure de la vitesse de courant sur le fond et de sa fluctuation, pour évaluer l'effet du courant de retour sur la mobilisation du fond,

- Détection et mesure de la géométrie du seuil chaque année ainsi que le volume de la fosse, afin de vérifier si l'augmentation du volume du seuil entraîne proportionnellement une augmentation du volume de la fosse,

- Mesure de la hauteur d'eau moyenne et la profondeur maximale de l'affouillement, chaque année afin de vérifier si la hauteur d'eau à un effet sur le creusement de la fosse,

- Des levés bathymétriques de part et d'autre de la fosse et dans tout le bras à différentes saisons, selon les conditions hydrologiques (débits, étiages, forts coefficients de marée et mortes eaux),

- Poursuite des campagnes de prélèvements du fond et des matières en suspension.

\section{5 - Conclusions}

A travers les différentes campagnes de mesures nous pouvons déduire qu'au cours des quatre dernières années, la fosse d'affouillement en aval du pont Anne de Bretagne s'est étendue et creusée. Ce demier est plus important au cours de l'hiver 94/95 en raison des crues de janvier à mars, de la présence d'une barge provoquant un retrécissement du bras et d'un bateau coulé dans l'axe de la pile située à proximité de la rive droite.

Il est probable que le lit de sable autour de cette pile ait migré, laissant à nu les fondations de la pile.

L'estimation de Breusers sur la profondeur maximale dû à un seuil noyé et l'estimation de la profondeur d'un affouillement associé à un retrécissement du lit semblent correctes.

Il faut à l'avenir parvenir à lier l'évolution de la fosse avec :

- le volume du seuil,

- l'intensité du courant de retour à l'intérieur de la fosse,

- le débit de l'écoulement,

- la cote moyenne de la ligne d'eau,

- la marée.

Ainsi sêra $t$-il possible de prévoir un schéma d'évolution générale de l'affouillement. Dans cette perspective, nous envisageons de poursuivre ces recherches et tenterons de développer une modélisation applicable à l'ensemble des ouvrages susceptibles d'êtres affouillés. 


\section{NOTATION ADOPTEE}

\begin{tabular}{|c|c|c|}
\hline ATON ADOPTEE & Symboles & Unités dans \\
\hline & & S.U.I \\
\hline Largeur de la rivière & $\mathrm{B}$ & $m$ \\
\hline Profondeur ou hauteur d'eau à partir du lit moyen & $H$ & $m$ \\
\hline Vitesse moyenne de l'écoulement & U & $\mathrm{m} / \mathrm{s}$ \\
\hline Vitesse critique d'entrainement du sédiment & $U_{C}$ & $\mathrm{~m} / \mathrm{s}$ \\
\hline Diamètre médian du grain (diamètre du sédiment à 50\%) & $d$ & $\mathrm{~m}$ \\
\hline Débit de la rivière & $\mathrm{Q}$ & $\mathrm{m}^{3} / \mathrm{s}$ \\
\hline Débit réduit & $q$ & $\mathrm{~m}^{2} / \mathrm{s}$ \\
\hline $\begin{array}{l}\text { Accélération de la pesanteur } \\
\text { Profondeur max. de l'affouillement par rapport au lit moyen naturel }\end{array}$ & $\begin{array}{l}g \\
d_{S}\end{array}$ & $m / s^{2}$ \\
\hline $\begin{array}{l}\text { Epaisseur du lit mobilisé à partir du lit moyen } \\
\text { Coefficient de frottement } \\
\text { Coefficient de forme }\end{array}$ & $\begin{array}{l}e^{2} \\
i \\
c_{f}\end{array}$ & $\mathrm{~m}$ \\
\hline Densité apparente du sédiment & $\delta$ & \\
\hline Débit solide & $q_{s}$ & $\mathrm{~m}^{2} / \mathrm{s}$ \\
\hline Largeur maximale de la fosse d'affouillement & is & m \\
\hline Longueur maximale de la fosse d'affouillement & $L_{s}$ & $\mathrm{~m}$ \\
\hline Volume de la fosse d'affoullement considérée comme elliptique & $V_{s}$ & $m^{3}$ \\
\hline Temps & t & \\
\hline
\end{tabular}

\section{BIBLIOGRAPHIE}

(1) S. ANDRE, Etude des affouillements dus soi soumis à un courant d'eau, Mémoire de DEÁ de Gérie civil, Génie côtiex, soutenu au laboratoire de mécanique et géomécanique de Nantes - Juillet 95

(2) C.I. BAKER, Theorelical approach to prediction of local scour around bridge piers, Journal of hydraulic research - Vol is . $1980 \mathrm{~N}^{\circ} 1$

(3) H.N.C. BREUSERS, G. NICOLLET, H.W. SHEN, Local scow around cylindrical piers., Joumal of Hydraulic research - Vol 15 1977

(4) H.N.C. BREUSERS, A.J. RAUDKIVI, Scouring, Hydraulic structures design manuel - 1991

(5) P. FATTAL, M. SANCHEZ, V. FREGARD, A. GROVEL, Y. DELANOE, Eude du bras de la Madeleine en aval du pont A. de Bretagne : élargissement du bras et évolution des fonds, Rapport $93-003$ du Centre Français du Littoral. 1993

(6) P. FATTAL, M. SANCHEZ, A. GROVEL, Y. DEL ANOE, Problèmes d'un estucire aménagé : la Loire, vers une recherche de solutions concertées, Actes des Jèmes Joumées Nationales Génie Civil, Génie Côtier, Sète, 2-4 mars 1994 - p. 127 - 132.

(7) P. FATTAL, M. ROBIr, Cinématique du lit de la Loire en aval du pont Anne de Brelagne (Nantes) traitee par modelisation numérique de terrain, Colıoque URA. 904. 1995,1996, à paraître

(8) 9. FATTAL, M. SANCHEZ, Y. DELANOE, Comportement hydraulique de devx bras de lo Loire (Pirmil et Madeleine) à Nantes, ; Actes des 4èmes Journéer Nationales Génie Civil, Gënie Côtier, Dinard, 17-19 avril 1996

(9) IZARD, BRADLEY, Field Verification of model tests on fiows through higway bridges and culverts, 7 th Hydraulic conference . Towa -1958

(10) J.P. LEVILLAIN,Conséquences de la surexploitation des scables de Loire... Comité Français de Géologie de lingénieur - Mai 1980

(11) I.P. LEYTLLAIN, Dimensionnement des protections contre les affouiliements, Joumées de formation en hydraulique fluviale Novernbre 86

(12) J.P. LEVILLAIN,Théorie et méthodes de détermination des affouillements, Joumées nationales Génie civil, Génie côtier - Actes dn collogue - pp 331 - 366 Février 92.

(13) J.P. LEVILLAN, Protection des sols autow des fondations d'un ouvrage d'art implanté sur lo Loire à Nantes, FONDACONCEPT - colloge intemational - Paris - Avil 94

(14) M. RAMETTE, Guide ähydraulique fluviale, Rapport INH - HE 40/81/04

(15) S. TRAN VAN THONG, Comparaison des données hydrologiques entre le bros de Pirmil et de la Madeleine et localisation du bouchon vaseux en 1995. Mémoire de DEA de Génie civil. Gérie côtier, soutenu au laboratoire de mécanique et géomécarique de Nantes - Juilles 95 